\title{
La prueba de esfuerzo electrocardiográfica: utilidad en el diagnóstico y pronóstico de la enfermedad coronaria
}

\author{
Exercise electrocardiogram testing: usefulness \\ in the diagnosis and prognosis of coronary artery disease
}

Jaime A. Gallo-Villegas PhD', J. Dagnovar Aristizábal-Ocampo MD²

\begin{abstract}
Resumen: La prueba de esfuerzo electrocardiográfica evalúa los ajustes fisiológicos en respuesta a un aumento en el metabolismo; además, permite establecer el diagnóstico y pronóstico de diferentes enfermedades cardiovasculares. Esta prueba continúa siendo el método diagnóstico de más fácil acceso y menos costoso para la evaluación inicial de pacientes con síntomas estables, que tienen un riesgo bajo o intermedio de enfermedad coronaria, y en aquellas personas con dolor torácico que consultan al servicio de urgencias. Sin embargo, posee limitaciones en la exactitud diagnóstica, con falsos negativos alrededor del $40 \%$ (sensibilidad del 60\%) y falsos positivos alrededor del 10\% (especificidad del 90\%). En este artículo se discute información sobre la prueba de esfuerzo electrocardiográfica, su aplicación tanto para el diagnóstico como el pronóstico y su utilidad en la toma de decisiones en los pacientes en quienes se sospecha o tienen una enfermedad coronaria. Igualmente, se presentan aspectos prácticos para la realización del procedimiento, una propuesta de informe con los principales hallazgos y algunos ejemplos clínicos.
\end{abstract}

Palabras clave: dolor en el pecho, enfermedad coronaria, prueba de esfuerzo, técnicas de diagnóstico cardiovascular.

Abstract: Exercise electrocardiography (exercise ECG) evaluates the physiological adjustments in response to an increased metabolism and allows the diagnosis and prognosis of various cardiovascular diseases. Exercise ECG remains the diagnostic test least expensive and most readily available for initial evaluation of patients with stable symptoms with low or intermediate risk of coronary heart disease and those consulting with chest pain to emergency services. However, it has limitations in diagnostic accuracy with false negatives rate around $40 \%$ (sensitivity $60 \%$ ) and false positives rate around 10\% (specificity 90\%). Here we discuss the information about the exercise ECG, its application for diagnosis and prognosis, and its use in decision-making for patients with suspected or known

\footnotetext{
'Médico, especialista en Medicina Aplicada a la Actividad Física y al Deporte, MSc en Epidemiología, candidato a PhD en Epidemiología, Centro Clínico y de Investigación SICOR, Soluciones Integrales en Riesgo Cardiovascular. Medellín, Colombia.

${ }^{2}$ Médico, cardiólogo, especialista en Ecocardiografia y Enfermedades Hipertensivas. Director científico, Centro Clínico y de Investigación SICOR, Soluciones Integrales en Riesgo Cardiovascular. Medellín, Colombia. Correspondencia: Calle 19 No 42-40. Teléfono: (057) (4) 6040007. Correo electrónico: dagnovar@une.netco
}

Conflicto de intereses: los autores declaran que no tienen conflicto de intereses Medicina \& Laboratorio 2015; 21: 63-84

Módulo 14 (Tecnología), número 20. Editora Médica Colombiana S.A. $2015^{\odot}$

Recibido el 27 de enero de 2015; aceptado el 12 de febrero de 2015 
coronary artery disease. In addition, practical aspects are presented to perform the procedure and a proposal of report with the main findings and some clinical examples.

Key words: chest pain, coronary disease, exercise test, cardiovascular diagnostic techniques.

Gallo-Villegas JA, Aristizábal-Ocampo JD. La prueba de esfuerzo electrocardiográfica: utilidad en el diagnóstico y pronóstico de la enfermedad coronaria. Medicina \& Laboratorio 2015; 21: 63-84

L a prueba de esfuerzo electrocardiográfica es el estudio diagnóstico más ampliamente utilizado para la evaluación de pacientes con sospecha de enfermedad coronaria. Además, puede ser usada para descartar o confirmar la presencia de isquemia, evaluar la severidad de la enfermedad coronaria, la capacidad de ejercicio y proveer información sobre el pronóstico en individuos sintomáticos $[1,2]$.

La incorporación de la prueba de esfuerzo electrocardiográfica en la evaluación de individuos con sospecha clínica de enfermedad coronaria se basa en la observación empírica de la depresión del segmento ST (intervalo que va desde el final del complejo QRS hasta el inicio de la onda T) en el electrocardiograma de pacientes con episodios de angina. Bousfield, en 1918, fue el primero en registrar la depresión del segmento ST en tres derivaciones electrocardiográficas en un paciente con angina [3]. Posteriormente, Feil y Siegel, en 1928, demostraron que los cambios en el segmento ST y la onda T (período de recuperación o repolarización de los ventrículos) durante el ejercicio podrían estar relacionados con isquemia miocárdica [4].

En una prueba de esfuerzo electrocardiográfica el ejercicio se utiliza de manera sistemática para observar los cambios electrocardiográficos y evaluar los ajustes fisiológicos en respuesta a diferentes demandas metabólicas que exceden los requerimientos en reposo [I]. Aunque inicialmente fue usada para la detección de isquemia en el electrocardiograma, actualmente tiene otras indicaciones como son: el diagnóstico y pronóstico de diferentes enfermedades cardiovasculares, evaluar la efectividad de intervenciones terapéuticas, detectar alteraciones en la respuesta del sistema cardio-respiratorio y definir la capacidad aeróbica funcional para una adecuada prescripción del ejercicio [1,5-9].

Debido a que la prueba de esfuerzo electrocardiográfica tiene limitaciones en la exactitud y seguridad diagnóstica para la detección de isquemia en pacientes con síntomas, antecedentes de enfermedad coronaria o algunas alteraciones en el electrocardiograma en reposo [ $1,2,5]$, en algunas ocasiones se recomienda complementar la evaluación con la utilización de técnicas de imagen como la ecocardiografía, los métodos de medicina nuclear y la tomografía para valorar alteraciones en la contractilidad, defectos en la perfusión miocárdica y la anatomía coronaria. Estas técnicas de imagen, combinadas con la prueba de esfuerzo electrocardiográfica convencional, permiten mejorar la capacidad diagnóstica y ayudan a localizar las arterias coronarias comprometidas [I,2].

La prueba de esfuerzo electrocardiográfica, a pesar de sus limitaciones, continúa siendo el método diagnóstico de más fácil acceso y menos costoso para la evaluación inicial de pacientes con síntomas relacionados con la enfermedad coronaria y que a su vez puede tener implicaciones en el pronóstico [1,2,5,6,8]. El objetivo de este artículo es presentar una revisión de la literatura actualizada sobre la prueba de esfuerzo electrocardiográfica y su utilidad práctica en el 
diagnóstico y pronóstico de los pacientes con sospecha de enfermedad coronaria. Inicialmente, se realiza una descripción general sobre el procedimiento, las recomendaciones, indicaciones y contraindicaciones; posteriormente, se hace énfasis en los aspectos más relevantes a tener en cuenta en la interpretación respecto al diagnóstico y el pronóstico; luego se describirá la utilidad de la prueba de esfuerzo electrocardiográfica en el servicio de urgencias; y, finalmente, a manera de ejemplo, se presentarán casos clínicos y un formato práctico que incluye la información más importante que se debe reportar luego del procedimiento.

\section{Procedimientos para la realización de la prueba de esfuerzo electrocardiográfica}

En 20I3, la Asociación Americana del Corazón (AHA; del inglés, American Heart Association) y el Colegio Americano de Cardiología (ACC; del inglés American College of Cardiology) presentaron las últimas guías sobre el procedimiento, las recomendaciones, indicaciones, contraindicaciones e interpretación de la prueba de esfuerzo electrocardiográfica en el escenario clínico [I]. Ese mismo año, la Sociedad Europea de Cardiología (ESC; del inglés, European Society of Cardiology) publicó las guías para el diagnóstico y tratamiento de los pacientes con enfermedad coronaria estable [2]. Estas recomendaciones serán consideradas en el desarrollo de la presente revisión.

La prueba de esfuerzo electrocardiográfica es un procedimiento seguro; sin embargo, se pueden presentar complicaciones como el infarto del miocardio o la muerte, con una frecuencia de uno en 2.500 pruebas [5]. Aunque las complicaciones son poco frecuentes, la prueba debe ser realizada por un médico entrenado en reanimación cardio-pulmonar, que conozca las indicaciones, contraindicaciones y los criterios para dar por terminada la prueba; además, que sea la persona responsable de la interpretación de los síntomas, los signos clínicos y el electrocardiograma [10]. Ante la posibilidad de complicaciones es necesario disponer durante la prueba de una camilla, oxígeno, un cardio-desfibrilador, medicamentos y otros implementos indispensables para una adecuada reanimación cardio-pulmonar en caso de que sea necesario. Previamente, se debe instruir al paciente para que no consuma café, alcohol ni cigarrillo tres horas antes de la realización de la prueba. Además, que evite la realización de actividad física intensa 12 horas antes del procedimiento, y que use ropa y calzado adecuado durante este.

Para la realización de la prueba de esfuerzo electrocardiográfica se pueden usar diferentes ergómetros; no obstante, la bicicleta y la banda rodante son los más utilizados. Durante las diferentes etapas de la prueba de esfuerzo electrocardiográfica se recomienda una monitorización y registro continuo del electrocardiograma, la frecuencia cardíaca, la presión arterial, la percepción del esfuerzo (escala de Borg) y los signos y síntomas [ I ]. Debido a la dificultad práctica para medir directamente el consumo de oxígeno $\left(\mathrm{VO}_{2}\right)$ máximo en una prueba de esfuerzo electrocardiográfica, es posible expresarlo en el equivalente metabólico basal (METs) a partir de la carga de trabajo máxima alcanzada, según el protocolo utilizado (Bruce, Balke, Naughton, Ellestad, entre otros) [I]. Actualmente se dispone de fórmulas de regresión lineal que permiten estimar el consumo de oxígeno máximo a partir de varios protocolos, validadas en diferentes poblaciones de estudio [I]. El protocolo más usado es el de Bruce en banda rodante, el cual especifica la velocidad, el porcentaje de inclinación, la duración y el número de las etapas de ejercicio [I] (véase tabla I). 
Tabla I. Recomendaciones para la realización de la prueba de esfuerzo electrocardiográfica

Lista de chequeo antes de iniciar la prueba de esfuerzo electrocardiográfica

I. Compruebe el funcionamiento del equipo (monitorización del electrocardiograma, banda rodante, reanimación cardiopulmonar)

2. ¿Cuál es la indicación de la prueba? (ver a continuación)

3. Compruebe que el paciente no tiene contraindicaciones para realizar la prueba (ver a continuación)

4. ¿La condición clínica del paciente ha cambiado desde la remisión?

5. ¿Cuáles medicamentos está tomando?

6. Evalúe la presión arterial

7. Compruebe que el electrocardiograma en reposo no ha cambiado desde la remisión

8. Explique al paciente cómo es el procedimiento y obtenga el consentimiento informado de forma escrita

Indicaciones de la prueba de esfuerzo electrocardiográfica

I. Estudio del dolor torácico en pacientes con una probabilidad pretest intermedia de enfermedad coronaria

2. Estratificación del riesgo en pacientes con enfermedad coronaria probable o conocida, como primera evaluación para determinar aquellos que tienen alto riesgo y se les debe realizar una coronariografía; además, pacientes que presentan cambios en el estado clínico

3. Evaluación del pronóstico después de un infarto del miocardio, para la prescripción del ejercicio, la incorporación a un programa de rehabilitación cardíaca y la valoración de la terapia médica

4. Posrevascularización miocárdica (angioplastia coronaria o cirugía de bypass coronario) si reaparecen síntomas que sugieren isquemia y para la prescripción del ejercicio o incorporación a un programa de rehabilitación cardíaca

5. Evaluación de personas diabéticas asintomáticas antes de iniciar un programa de ejercicio

Contraindicaciones de la prueba de esfuerzo electrocardiográfica

I. Síndrome coronario agudo (dentro de los dos días siguientes)

2. Arritmia cardíaca no controlada

3. Estenosis aórtica severa sintomática

4. Insuficiencia cardíaca descompensada

5. Embolia pulmonar aguda

6. Miocarditis o pericarditis aguda

7. Disección aórtica aguda

8. Enfermedad sistémica aguda

9. Presión arterial sistólica mayor a $200 \mathrm{mmHg}$ o diastólica mayor a $110 \mathrm{mmHg}$

10. Aumento en el riesgo de caídas debido a trastornos neurológicos o musculoesqueléticos

Finalización de la prueba de esfuerzo electrocardiográfica:

I. Limitación por los síntomas o solicitud del paciente a terminarla

2. Alcanzar la frecuencia cardíaca objetivo

3. Depresión del segmento ST $>2 \mathrm{~mm}$ (horizontal o descendente)

4. Elevación del segmento $\mathrm{ST}>2 \mathrm{~mm}$ en derivaciones, sin ondas $\mathrm{Q}$ patológicas

5. Arritmia sostenida

6. Caída en la presión arterial sistólica $>10 \mathrm{mmHg}$ con evidencia de isquemia miocárdica o bajo gasto cardíaco

7. Aumento de la presión arterial sistólica $>250 \mathrm{mmHg}$

8. Dificultades técnicas que impidan la monitorización del electrocardiograma o de la presión arterial

Tomado y adaptado de "Exercise standards for testing and training: a scientific statement from the American Heart Association" por GF Fletcher y colaboradores, 2013, Circulation, 128, p. 873-934. Copyright ${ }^{\odot} 2015$ por American Heart Association, Inc; $y$ "2013 ESC guidelines on the management of stable coronary artery disease: the Task Force on the management of stable coronary artery disease of the European Society of Cardiology" por G Montalescoty colaboradores, 2013, Eur Heart J, 34, p. 2949-3003. Copyright ${ }^{\odot} 2015$ por European Society of Cardiology

\section{La prueba de esfuerzo electrocardiográfica y la enfermedad coronaria}

Diferentes pruebas pueden ser usadas para el diagnóstico de la enfermedad coronaria. Dichas pruebas se pueden clasificar en dos tipos: a) aquellas que muestran la anatomía de las arterias coronarias epicárdicas y b) aquellas que revelan los efectos funcionales de la enfermedad coronaria. En presencia de enfermedad coronaria en la que las placas de ateroma limitan el flujo sanguíneo la perfusión miocárdica es insuficiente para satisfacer las demandas metabólicas durante el ejercicio, lo que resulta en isquemia subendocárdica y alteraciones en la repolarización 
ventricular, que son evidenciadas en el electrocardiograma como una depresión del segmento ST (véase figura I). La prueba de esfuerzo electrocardiográfica, además de la capacidad para demostrar la isquemia miocárdica inducible y la respuesta hemodinámica al ejercicio, puede ayudar a esclarecer las causas de las manifestaciones clínicas no específicas relacionados con el esfuerzo físico y proporcionar una medida objetiva de la tolerancia al ejercicio y los síntomas referidos por el paciente [6].
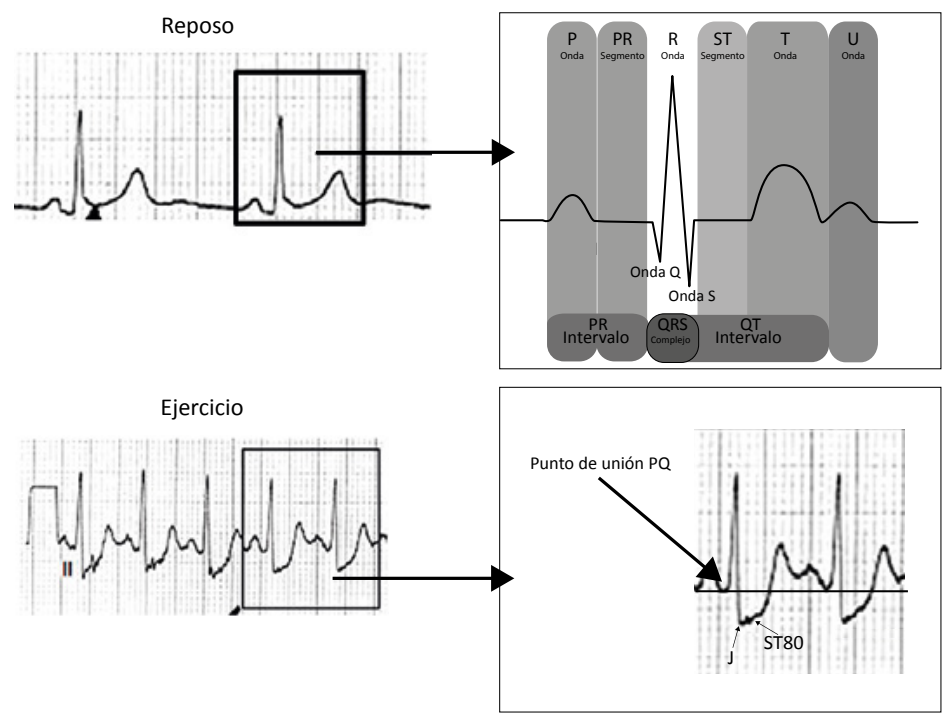

Figura I. Prueba de esfuerzo electrocardiográfica de un hombre de 70 años remitido para evaluar la presencia de angina estable. En el electrocardiograma en reposo se señala una elevación del segmento ST de 0,9 mm que indica una repolarización temprana. El electrocardiograma de ejercicio muestra una depresión del segmento ST de 2,5 mm 80 ms después del punto J (ST80). La unión PQ representa la línea de base. La prueba fue finalizada por la aparición de angina típica a una frecuencia cardíaca de 135 latidos/min. Los cambios del segmento ST durante el ejercicio representan una respuesta isquémica en un paciente sintomático con una probabilidad pretest intermedia o alta.

\section{Interpretación de la prueba de esfuerzo electrocardiográfica}

La prueba de esfuerzo electrocardiográfica se debe reportar como positiva si aparece alguna de las siguientes características: alteraciones electrocardiográficas (depresión o elevación del segmento ST) o dolor en el tórax (angina) [7].

\section{Alteraciones electrocardiográficas}

Un ciclo cardíaco está representado por una sucesión de ondas en el trazado del electrocardiograma: la onda P, el complejo QRS, la onda T y la onda U, con dos segmentos el PR, y el ST, y dos intervalos, el PR y el QT (véase figura I). Durante el ejercicio, en sujetos normales, ocurren diferentes cambios en prácticamente todos los componentes de un electrocardiograma, los cuales incluyen el acortamiento progresivo de los intervalos. La amplitud de la onda P aumenta, pero la amplitud de la onda $T$ disminuye. Igualmente, ocurre una desviación progresiva del eje del QRS a la derecha y la amplitud de la onda R cambia. El punto J (unión entre el complejo QRS y el segmento ST) desciende sobre todo en personas de edad avanzada, pero el segmento ST es rápidamente ascendente. 
Uno de los criterios para considerar una prueba de esfuerzo electrocardiográfica positiva es la depresión del segmento ST durante o después del ejercicio mayor o igual que $0, I \mathrm{mV}(\mathrm{I} \mathrm{mm})$ horizontal o en descenso con un segmento PR que es isoeléctrico con relación a la línea de base (antes de la onda P) del electrocardiograma. La depresión del segmento ST se mide 80 ms después del punto I y debe estar presente al menos en tres latidos consecutivos y en dos o más derivaciones electrocardiográficas contiguas (véase figura I).

Es importante resaltar que entre mayor sea la depresión del segmento ST mayor es la probabilidad de enfermedad coronaria. Una depresión del segmento ST en descenso es un predictor más potente que la depresión del segmento ST horizontal (plana), y ambas son más predictivas que la depresión del segmento ST en ascenso (véase figura 2). En general, una depresión del segmento ST rápida, es decir, que regresa a la línea de base en el primer minuto de la recuperación, se asocia con enfermedad coronaria menos severa y con menos anormalidades de perfusión en comparación con pacientes que tienen una recuperación más lenta de la depresión del segmento ST [I I].
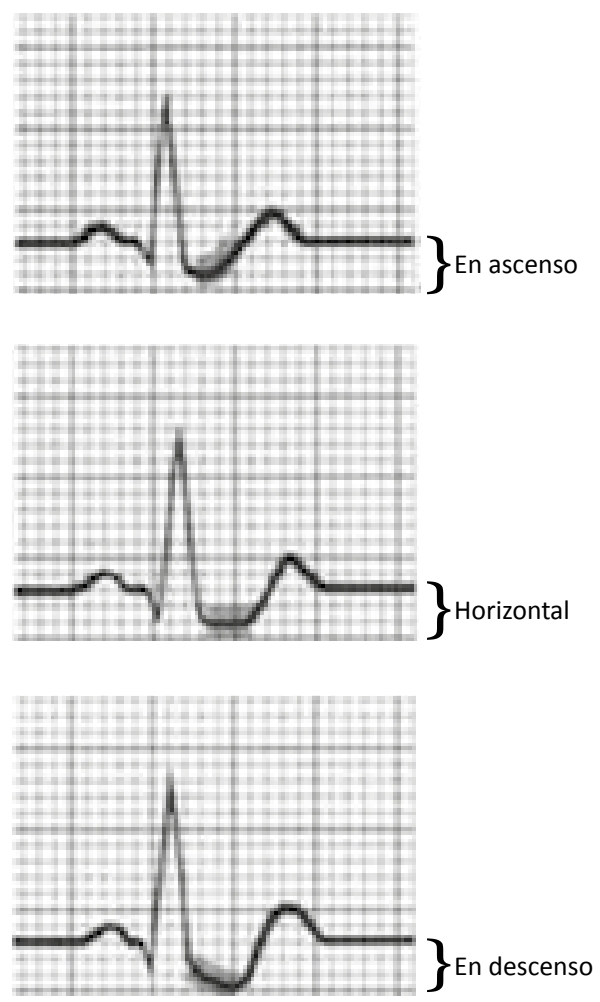

Figura 2. Tipos de depresión del segmento ST en ascenso, horizontal y en descenso.
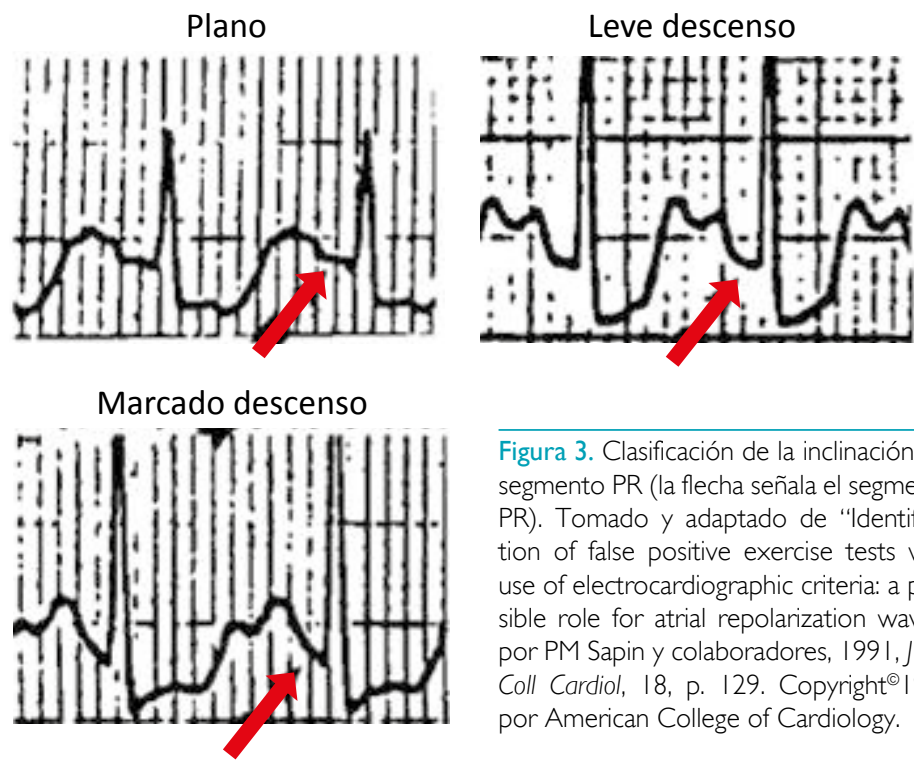

Figura 3. Clasificación de la inclinación del
segmento PR (la flecha señala el segmento
PR). Tomado y adaptado de "Identifica-
tion of false positive exercise tests with
use of electrocardiographic criteria: a pos-
sible role for atrial repolarization waves"
por PM Sapin y colaboradores, I99|,J Am
Coll Cardiol, I8, p. I29. Copyright 199 |
por American College of Cardiology. 
La depresión del segmento ST causada por enfermedad coronaria es vista más frecuentemente en las derivaciones unipolares precordiales $\mathrm{VI}$ y $\mathrm{V} 5$. La depresión del ST en las derivaciones de la cara inferior debe ser interpretada con cuidado pues es frecuente que se deba a falsos positivos provocados principalmente por un descenso marcado del segmento PR en esas derivaciones (véase figuras 3 y 4) [12]. Las derivaciones en las cuales se produce la depresión del segmento ST no señalan la localización exacta de la región isquémica ni las arterias coronarias comprometidas [7]. Los posibles mecanismos involucrados en la depresión del segmento ST en el electrocardiograma incluyen cambios en el potencial de membrana de las células del miocardio producidos por modificaciones en la duración del potencial de acción en la región isquémica, así como un gradiente espacial del potencial de membrana de reposo entre la región isquémica y la no isquémica [6].

Otro criterio de positividad que también puede ocurrir, pero con menor frecuencia, es el ascenso del segmento ST igual o mayor a I mm que en ausencia de un infarto del miocardio previo puede representar un vasoespasmo coronario

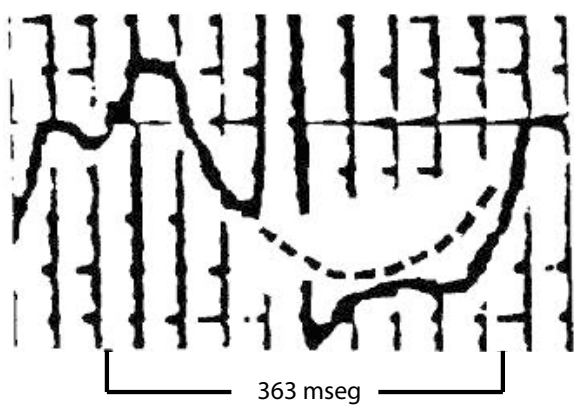

Figura 4. Derivación II del electrocardiograma que muestra una marcada onda de repolarización atrial que influye en la interpretación del segmento ST y puede llevar a un falso positivo de la prueba de esfuerzo electrocardiográfica. Tomado y adaptado de "Identification of false positive exercise tests with use of electrocardiographic criteria: a possible role for atrial repolarization waves" por PM Sapin y colaboradores, 1991, J Am Coll Cardiol, I8, p. 133. Copyright (C)1991 por American College of Cardiology.
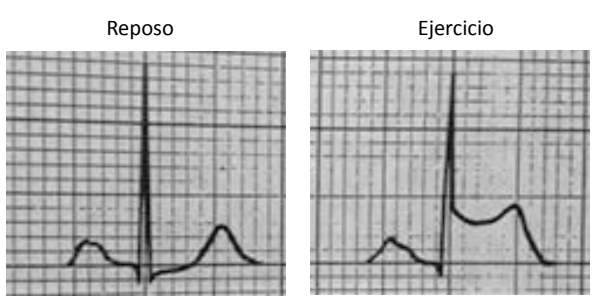

Figura 5. Elevación del punto I y del segmento ST que indica isquemia transmural grave causada por una enfermedad coronaria severa o espasmo coronario. o enfermedad coronaria grave. La elevación del segmento ST debe ser mayor o igual que $0,1 \mathrm{mV}(\mathrm{I} \mathrm{mm})$ horizontal o en ascenso, medido 80 ms después del punto J y que se observa en tres o más latidos consecutivos (véase figura 5). Esta elevación es indicativa de una isquemia transmural grave causada por una enfermedad coronaria severa o un espasmo coronario observado en la angina variante o de Prinzmetal [7].

Una elevación del segmento ST en las derivaciones aVR (del inglés, augmented vector right) puede ser vista cuando hay un compromiso severo en el flujo del tronco coronario izquierdo $y$, a diferencia de la depresión del segmento ST, la elevación sí localiza la región isquémica y señala un mayor territorio isquémico [7]. En una derivación que presenta ondas $Q$ desde el reposo la elevación del segmento puede reflejar alteraciones en el movimiento de la pared por un aneurisma y está asociado con acinesia o disquinesia de la pared del ventrículo izquierdo, viabilidad residual de una zona que presentó un infarto o isquemia periinfarto (véase figura 6) [13].

\section{Dolor torácico}

Otro criterio de positividad de la prueba de esfuerzo electrocardiográfica es el dolor en el pecho de características anginosas que se inicia durante o después del ejercicio y que puede ser denominado angina o equivalente anginoso. De los pacientes con una prueba de esfuerzo electrocardiográfica positiva, la mayoría presentan cambios en el segmento ST mientras que sólo 
un tercio desarrollan angina de pecho [7]. La presentación de angina típica durante la prueba de esfuerzo electrocardiográfica se relaciona con isquemia y enfermedad coronaria más severa [7]. En los pacientes diabéticos la aparición de dolor es menos frecuente [14].

\section{Otras alteraciones que pueden aparecer durante la prueba de esfuerzo electrocardiográfica}

Durante la prueba de esfuerzo electrocardiográfica también se pueden observar otras alteraciones en la respuesta hemodinámica durante el ejercicio que se caracterizan por cambios en la frecuencia cardíaca o la presión arterial [7]. Se puede presentar incompetencia cronotrópica, un incremento rápido de la frecuencia cardíaca a bajas intensidades, una lenta recuperación de la frecuencia cardíaca luego del esfuerzo físico, una baja respuesta de la presión arterial sistólica o incluso hipotensión, y una respuesta hipertensiva durante el ejercicio. Dichas alteraciones hemodinámicas pueden estar relacionadas con diferentes condiciones clínicas y deberían reportarse en el informe final del procedimiento [I,7].

\section{Utilidad de la prueba de esfuerzo electrocardiográfica en el diagnóstico} de la enfermedad coronaria estable

Las pruebas diagnósticas son un componente esencial en la práctica clínica; no solamente para el diagnóstico, como su nombre lo indica, sino también para la toma de decisiones relacionadas con el pronóstico [15]. La evaluación de la probabilidad de que la enfermedad coronaria sea la causa del dolor en el tórax inicia con una historia clínica cuidadosa, un examen físico y el análisis del electrocardiograma en reposo. Teniendo en cuenta las características de los síntomas, la edad y el sexo es posible calcular la probabilidad pretest de la enfermedad coronaria (véase tabla 2), la cual puede orientar la selección del método diagnóstico [2,16]. En situaciones clínicas de prevalencia alta (p. ej. probabilidad pretest alta, es decir, mayor del 85\%) o baja (menor del I5\%) usualmente 


\begin{tabular}{|c|c|c|c|c|c|c|}
\hline \multirow[t]{2}{*}{ Edad } & \multicolumn{2}{|c|}{ Angina típica } & \multicolumn{2}{|c|}{ Angina atípica } & \multicolumn{2}{|c|}{ Dolor no anginoso } \\
\hline & Hombres & Mujeres & Hombres & Mujeres & Hombres & Mujeres \\
\hline $30-39$ & 59 & 28 & 29 & 10 & 18 & 5 \\
\hline $40-49$ & 69 & 37 & 38 & 14 & 25 & 8 \\
\hline $50-59$ & 77 & 47 & 49 & 20 & 34 & 12 \\
\hline $60-69$ & 84 & 58 & 59 & 28 & 44 & 17 \\
\hline $70-79$ & 89 & 68 & 69 & 37 & 54 & 24 \\
\hline$>80$ & 93 & 76 & 78 & 47 & 65 & 32 \\
\hline
\end{tabular}

Las probabilidades de enfermedad coronaria que se muestran reflejan los cálculos para los pacientes con 35, 45 ,

$55,65,75$ y 85 años de edad.

Tomado y adaptado de "2013 ESC guidelines on the management of stable coronary artery disease: the Task Force on the management of stable coronary artery disease of the European Society of Cardiology" por G Montalescot y colaboradores, 2013 , Eur Heart J, 34, p. 2962. Copyright ${ }^{\circledR} 2015$ por European Society of Cardiology

las decisiones no se modifican por el resultado de ninguna prueba, aunque ésta sea de un óptimo desempeño.

Cuando la probabilidad pretest de enfermedad coronaria es muy baja (menor del 15\%) se debe buscar otra causa de dolor torácico y no se debe utilizar ningún método diagnóstico, mientras que en quienes la probabilidad pretest de enfermedad coronaria es muy alta (mayor del 85\%), por ejemplo, en hombres de 75 años o más con angina típica, tampoco es necesario realizar exámenes diagnósticos debido a que no va a ser posible mejorar la exactitud diagnóstica. En este sentido, los métodos diagnósticos tiene una mayor utilidad en el área de mayor incertidumbre diagnóstica, cuando la probabilidad pretest de la enfermedad coronaria es intermedia; es decir, entre el $15 \%$ y el $85 \%$, donde son útiles precisamente para aumentar la exactitud diagnóstica y llevar esa probabilidad pretest a una probabilidad postest que permita tomar decisiones clínicas respecto a la presencia o ausencia de la enfermedad coronaria y guiar el tratamiento médico.

Las características operativas tradicionales de las pruebas diagnósticas (sensibilidad, especificidad y valores predictivos) tienen algunas limitaciones para su uso en la práctica clínica. Aunque en ocasiones se utiliza sólo la sensibilidad y la especificidad de una prueba para evaluar su utilidad, estos valores no brindan la información suficiente para tomar una adecuada decisión clínica. Teniendo en cuenta sólo la sensibilidad o especificidad de una prueba es imposible concluir si un paciente tiene o no una enfermedad. La interpretación del resultado de una prueba, en el contexto del diagnóstico, requiere del uso de los valores predictivos positivos y negativos que a su vez cambian, dependiendo del escenario clínico, con la prevalencia o probabilidad previa de la enfermedad (probabilidad pretest) (véase tabla 2).

Los estudios que han sido publicados sobre la capacidad diagnóstica de la prueba de esfuerzo electrocardiográfica han reportado una sensibilidad del 60\% (rango entre 45\% y 92\%) y una especificidad del 77\% (rango entre 17\% y 92\%)[5, I7]. La variabilidad reportada en la sensibilidad y especificidad puede ser explicada por los diferentes puntos de corte utilizados para definir la positividad, el hecho de ser realizados en diferentes poblaciones y la presencia de múltiples sesgos, debido a la omisión de algunas variables de confusión potenciales que pueden haber afectado la validez interna de los estudios [6]. 
Entre las variables de confusión potenciales se han descrito: la presencia de lesiones no obstructivas, pero vulnerables, la dificultad para alcanzar el umbral en la severidad de la isquemia ( $p$. ej. por una inadecuada tolerancia al esfuerzo o bloqueo en la respuesta de la frecuencia cardíaca al ejercicio), presencia de algún factor que aumenta el estrés de la pared del endocardio y limita la reserva en el flujo sanguíneo subendocárdico, el efecto de electrolitos o medicamentos que afectan el potencial de acción, así como áreas discontinuas de isquemia orientadas que suprimen los cambios en el segmento ST [6]. Cuando se define como positivo una prueba utilizando el criterio de una depresión del segmento ST mayor o igual que I mm horizontal o en descenso, la sensibilidad es del $60 \%$ y la especificidad del $90 \%$ [7].

Teniendo en cuenta las características operativas de la prueba de esfuerzo electrocardiográfica, la ausencia de depresión del segmento ST no excluye una enfermedad coronaria que limita el flujo. La prueba de esfuerzo electrocardiográfica tiene una frecuencia de falsos negativos alrededor del 40\% (sensibilidad del 60\%). Para disminuir estos valores se recomienda que en los casos en los que el paciente no alcance el $85 \%$ de la frecuencia cardíaca esperada para la edad y no experimente angina, o el electrocardiograma no muestre depresión del segmento ST, el resultado de la prueba se reporte como no concluyente en lugar de negativo. Los falsos negativos en la prueba de esfuerzo electrocardiográfica hacen referencia a la ausencia de cambios en el segmento ST (prueba negativa) en presencia de una estenosis significativa (mayor del 70\%) de una arteria coronaria epicárdica, los cuales se han asociados al género masculino, la presencia de enfermedad de un vaso, la imposibilidad para alcanzar una adecuada carga de trabajo físico, los medicamentos que limitan el trabajo miocárdico con el ejercicio o que mejoran el flujo sanguíneo miocárdico [7] (véase tabla 3).

La depresión del segmento ST durante la prueba de esfuerzo electrocardiográfica no confirma la presencia de enfermedad coronaria debido a que se puede presentar en personas sanas sin enfermedad cardíaca, más común en mujeres que hombres (falsos positivos alrededor del 10\%, teniendo en cuenta una especificidad del 90\%). Los falsos positivos en una prueba de esfuerzo electrocardiográfica hacen referencia a la presencia de una depresión del segmento ST en ausencia de una estenosis significativa (mayor del 70\%) de una arteria coronaria epicárdica, los cuales se encuentran asociados a situaciones que llevan a un incremento en la masa del ventrículo izquierdo, sobrecarga de presión o de volumen y aumento en los requerimientos de oxígeno que lleva a un desequilibrio entre la oferta y la demanda del flujo sanguíneo al subendocardio. También, se han encontrado falsos positivos relacionados con la utilización de algunos medicamentos como la digoxina, el aumento en la estimulación simpática, los bloqueos de rama, los síndromes de preexcitación, las alteraciones hidroelectrolíticas y los cambios metabólicos que afectan el segmento ST por incremento en la frecuencia cardíaca [7] (véase tabla 3).

\section{Interpretación de la prueba de esfuerzo electrocardiográfica con análisis de probabilidades}

La interpretación del resultado de una prueba de esfuerzo electrocardiográfica positiva o negativa (probabilidad de tener una enfermedad coronaria luego de realizar la prueba o proba- 


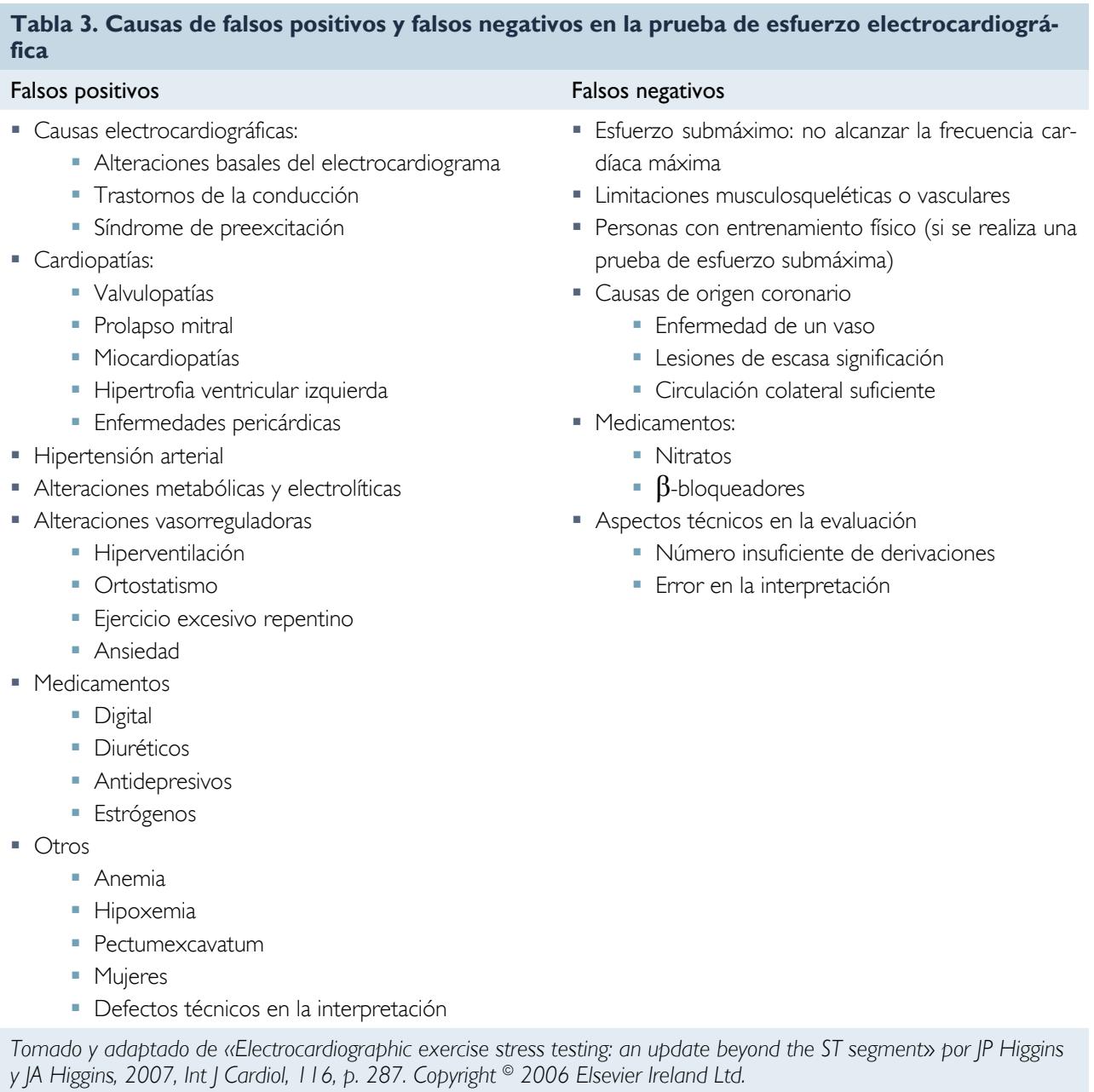

bilidad postest), de acuerdo a lo establecido en el teorema de Bayes, debe tener en cuenta la probabilidad pretest cuando el método no es completamente exacto. El teorema de Bayes, en la teoría de probabilidad, fue planteado por el filósofo inglés Thomas Bayes en 1763 [I8] para expresar la probabilidad condicional de un evento aleatorio A dado B. En términos generales, el teorema de Bayes es de enorme relevancia debido a que considera que la interpretación de la observación presente depende de la experiencia pasada.

En el caso de la prueba de esfuerzo electrocardiográfica, el teorema de Bayes permite relacionar la probabilidad de la enfermedad en el paciente antes de que la prueba sea realizada (probabilidad pretest) con la probabilidad de la enfermedad después de la prueba (probabilidad postest). De esta manera, si la probabilidad pretest de enfermedad coronaria es del $10 \%$ y la información contenida en la prueba (capacidad de la prueba para incrementar la información) es del $50 \%$, es posible calcular la incertidumbre postest o, contrariamente, la probabilidad postest de enfermedad coronaria.

En la figura 7 se esquematiza el cálculo de la certeza diagnóstica a partir de la información contenida en la prueba (50\%), que conduce a una probabilidad postest del $60 \%$ o una incerti- 


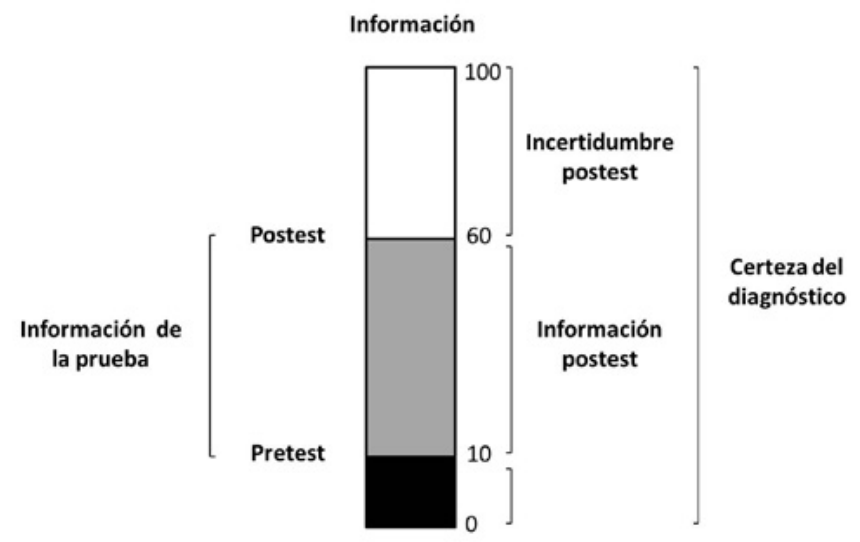

Figura 7. Teorema de Bayes. Asumiendo que la probabilidad pretest es del $10 \%$ y la información contenida en la prueba es del $50 \%$ es posible calcular la probabilidad postest, equivalente en este caso al $60 \%$, o la incertidumbre remanente, equivalente al $40 \%$. La información contenida varía con la naturaleza de la prueba y la prevalencia de la enfermedad en la población de estudio.

dumbre remanente del 40\%. Teniendo en cuenta el teorema de Bayes, según la probabilidad pretest de enfermedad coronaria, un resultado positivo por este método diagnóstico en un paciente de bajo riesgo (menor que el 15\%) puede ser en realidad un falso positivo y un resultado negativo por este método diagnóstico en un paciente de alto riesgo (mayor que el 85\%) puede ser en realidad un falso negativo.

La comparación del rendimiento diagnóstico entre la prueba de esfuerzo electrocardiográfica, la ecocardiografía de estrés con dobutamina, la tomografía computarizada por emisión de fotones (SPECT; del inglés, Single Photon Emission Computed Tomography) de ejercicio y la angiografía coronaria por tomografía computarizada (CTA; del inglés, Computed Tomography Angiography) se puede realizar utilizando las razones de probabilidad (véase tabla 4). Esta medida permite resumir el rendimiento diagnóstico de una prueba mediante la comparación de dos probabilidades: positiva y negativa.

\begin{tabular}{|c|c|c|c|c|}
\hline & $\begin{array}{l}\text { Prueba de esfuerzo } \\
\text { electrocardiográfica }\end{array}$ & $\begin{array}{l}\text { Ecocardiografía de } \\
\text { estrés con dobuta- } \\
\text { mina }\end{array}$ & $\begin{array}{l}\text { Tomografía compu- } \\
\text { tarizada por emisión } \\
\text { de fotones (SPECT) } \\
\text { con ejercicio }\end{array}$ & $\begin{array}{l}\text { Angiografía coro- } \\
\text { naria por tomogra- } \\
\text { fía computarizada } \\
\text { (CTA) }\end{array}$ \\
\hline $\begin{array}{l}\text { Verdaderos positivos } \\
\text { (sensibilidad) (\%) }\end{array}$ & 60 & 81 & 83 & 97 \\
\hline Falsos negativos (\%) & 40 & 19 & 17 & 3 \\
\hline $\begin{array}{l}\text { Verdaderos negati- } \\
\text { vos (especificidad) } \\
\text { (\%) }\end{array}$ & 90 & 84 & 75 & 74 \\
\hline Falsos positivos (\%) & 10 & 16 & 25 & 26 \\
\hline $\begin{array}{l}\text { Razón de probabili- } \\
\text { dades positiva }\end{array}$ & 6 & 5 & 3 & 4 \\
\hline $\begin{array}{l}\text { Razón de probabili- } \\
\text { dades negativa }\end{array}$ & 0,4 & 0,2 & 0,2 & 0,04 \\
\hline \multicolumn{5}{|c|}{$\begin{array}{l}\text { Tomado y adaptado de «2013 ESC guidelines on the management of stable coronary artery disease: the Task Force on } \\
\text { the management of stable coronary artery disease of the European Society of Cardiology» por G Montalescot y colabora- } \\
\text { dores, } 2013 \text {, Eur Heart J, 34, p. 2949-3003. Copyright } \odot 2015 \text { por European Society of Cardiology }\end{array}$} \\
\hline
\end{tabular}


En el caso de la enfermedad coronaria, una razón de probabilidad positiva representa la proporción de personas cuyo resultado es positivo y tienen la enfermedad en relación con la proporción de personas cuyo resultado es positivo pero no tienen la enfermedad, y es expresada como:

\section{razón de probabilidad positiva=sensibilidad/( 1 -especificidad $)$}

Por su parte, una razón de probabilidad negativa representa la proporción de personas cuyo resultado es negativo, pero tienen la enfermedad coronaria en relación con la proporción de personas cuyo resultado es negativo y no tienen la enfermedad coronaria, y es expresada como:

\section{razón de probabilidad negativa $=($ I-sensibilidad $) /$ especificidad}

Así, un resultado positivo por un método diagnóstico con una razón de probabilidades positiva mayor que 10 efectivamente señala la presencia de la enfermedad, mientras que un resultado negativo con los métodos diagnósticos con una razón de probabilidad negativa menor que 0,I efectivamente la descarta (véase tabla 5).

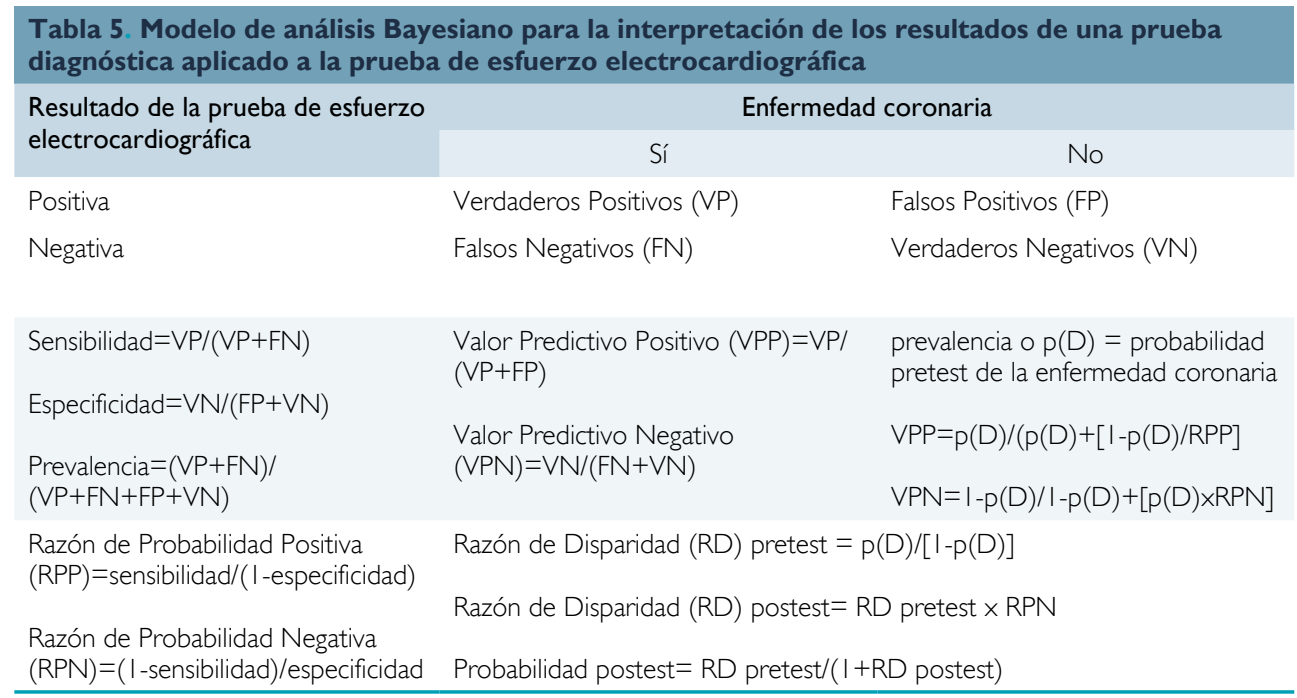

Como se observa en la información suministrada en la tabla 4 ninguno de los métodos diagnósticos comparados tiene la capacidad para confirmar la presencia de enfermedad coronaria y, por lo tanto, su principal aplicación es descartarla. De estos métodos diagnósticos, la angiografía coronaria por tomografía computarizada es el mejor para descartar la presencia de enfermedad coronaria debido a que tiene una razón de probabilidades negativa menor que 0, I, lo que indica que una angiografía coronaria por tomografía computarizada negativa excluye efectivamente la presencia de enfermedad coronaria. La ecocardiografía de estrés con dobutamina y la tomografía computarizada por emisión de fotones de ejercicio se encuentran en el segundo lugar como pruebas para descartar la presencia de enfermedad coronaria. Por su parte, la prueba de esfuerzo electrocardiográfica tiene la menor capacidad diagnóstica; sin embargo, continúa siendo útil en pacientes con una probabilidad pretest de enfermedad coronaria baja o intermedia.

Luego de realizar una prueba de esfuerzo electrocardiográfica es importante conocer cuál es la probabilidad de tener o no una enfermedad coronaria según el resultado de la prueba. Esta información se puede obtener a partir del cálculo de los valores predictivos positivos (VPP) y 
negativos (VPN), teniendo en cuenta la probabilidad pretest $(P(D))$ de la enfermedad coronaria y la información suministrada por la prueba en las razones de probabilidad positiva (RPP) y negativa (RPN), así:

$$
\begin{gathered}
V P P=p(D) /(P(D)+[I-p(D) / R P P] \\
V P N=I-p(D) / I-p(D)+[P(D) \times R P N]
\end{gathered}
$$

El valor predictivo positivo equivale a la probabilidad de encontrar la enfermedad coronaria entre los individuos en los cuales la prueba fue positiva, mientras que el valor predictivo negativo es igual a la probabilidad de no encontrar la enfermedad coronaria entre los individuos en los cuales la prueba fue negativa (véase tabla 5).

Si bien las razones de probabilidad de la prueba de esfuerzo electrocardiográfica se pueden utilizar en diferentes escenarios de probabilidad pretest de la enfermedad coronaria, es decir, el mismo valor de razón de probabilidad positiva o razón de probabilidad negativa de la prueba se puede aplicar sin modificaciones en situaciones con diferentes prevalencias, esto no significa que en la interpretación y el uso clínico de los valores de las razones de probabilidad se ignoren las características clínicas de cada paciente, que a su vez se relacionan con la probabilidad de tener la enfermedad coronaria. Este traslado de la probabilidad previa a la probabilidad posterior por medio de las razones de probabilidad requiere de una operación algebraica a partir del teorema de Bayes:

(probabilidad previa/[I-probabilidad previa $]$ ) $\mathrm{RP}=$ probabilidad posterior/(I-probabilidad posterior)

Este cálculo también se puede realizar de una forma más sencilla con la utilización de aplicaciones en la Web o el uso de un nomograma $[19,20]$. A partir de este nomograma con la probabilidad pretest de enfermedad coronaria y las razones de probabilidad positiva y negativa, según el resultado de la prueba de esfuerzo electrocardiográfica (razón de probabilidad positiva para una prueba positiva o razón de probabilidad negativa para una prueba negativa), es posible calcular la probabilidad postest de presentar la enfermedad coronaria (véase tabla 5).

\section{Recomendaciones para el uso de la prueba de esfuerzo electrocardiográfica}

Debido a las limitaciones diagnósticas de la prueba de esfuerzo electrocardiográfica, el Instituto Nacional de Excelencia para la Salud y los Cuidados (NICE; del inglés, National Institute for Health and Care Excellence), en sus guías publicadas en el 2010, indicó que la prueba de esfuerzo electrocardiográfica no es el método diagnóstico preferido en cualquier grupo de pacientes con sospecha de enfermedad coronaria estable y, en su lugar, la consideró una alternativa aceptable como prueba funcional no invasiva en pacientes que tienen de nuevo dolor torácico, en quienes ya se ha confirmado la presencia de enfermedad coronaria (p. ej. pacientes que ya han presentado un infarto de miocardio o se les haya realizado una cirugía de revascularización coronaria).

Por su parte, la Asociación Americana del Corazón y el Colegio Americano de Cardiología en 2012 [2 I] y la Sociedad Europea de Cardiología en 2013 [2] aprobaron el uso de la prueba de esfuerzo electrocardiográfica para el estudio de pacientes con dolor torácico con una probabilidad baja o intermedia de enfermedad coronaria, que tienen un electrocardiograma normal en reposo y son capaces de hacer ejercicio. Esta recomendación se fundamenta en el rendi- 
miento diagnóstico aceptable de la prueba de esfuerzo electrocardiográfica en esta población, junto con el bajo costo y amplia disponibilidad que la caracteriza. Además, hay pocos ensayos clínicos y evidencia limitada que demuestren que el uso de otros métodos diagnósticos con mayor capacidad diagnóstica confieran un mejor resultado [22]. En la figura 8 se presenta un flujograma con las recomendaciones de la Sociedad Europea de Cardiología [2] para el estudio de pacientes con dolor torácico en quienes se sospecha una enfermedad coronaria estable.

\begin{tabular}{|c|c|c|c|c|c|c|}
\hline \multicolumn{7}{|c|}{ Síndrome de dolor torácico estable } \\
\hline \multicolumn{7}{|c|}{ Evaluación clínica y electrocardiográfica } \\
\hline \multicolumn{7}{|c|}{ Determinación de la probabilidad de enfermedad coronaria según la historia del paciente, edad y sexo } \\
\hline \multicolumn{7}{|c|}{ Probabilidad pre-prueba de enfermedad coronaria } \\
\hline$<15 \%$ & \multicolumn{3}{|c|}{$15 \%-65 \%$} & \multicolumn{2}{|c|}{$66 \%-85 \%$} & $>85 \%$ \\
\hline $\begin{array}{l}\text { Considerar otras causas } \\
\text { de dolor torácico. } \\
\text { No realizar la prueba } \\
\text { diagnóstica }\end{array}$ & \multicolumn{3}{|c|}{$\begin{array}{c}\text { Prueba de esfuerzo electrocardiográfica: razonable como } \\
\text { primer método diagnósticosi el paciente puede hacer ejercicio, } \\
\text { tiene un electrocardiograma normal, y no se sospecha o conoce } \\
\text { deterioro de la función sistólica ventricular izquierda. } \\
\text { Prueba de estrés con imágenes: realizar en lugar de la prueba } \\
\text { de esfuerzo electrocardiográfica cuando no se cumplen los } \\
\text { criterios y es preferible si se cuenta con la capacidad y } \\
\text { experticia necesaria. } \\
\text { Angiografia coronaria por tomografia computarizada: preferible } \\
\text { en pacientes con probabilidad pre-prueba entre } 15 \% \text { y } 50 \% \text { si } \\
\text { se cuenta con la capacidady experticia necesaria. }\end{array}$} & \multicolumn{2}{|c|}{$\begin{array}{l}\text { Prueba de estrés con } \\
\text { imágenes: realizar como } \\
\text { primera prueba } \\
\text { diagnóstica }\end{array}$} & $\begin{array}{l}\text { Enfermedad coronaria } \\
\text { muy probable. } \\
\text { No se requiere más } \\
\text { pruebas diagnósticas. } \\
\text { Iniciar terapia médica } \\
\text { óptima. } \\
\text { Ordenar una prueba } \\
\text { pronóstica. } \\
\text { Realizar una angiografía } \\
\text { coronaria si persisten } \\
\text { los síntomas o el } \\
\text { pronóstico es de alto } \\
\text { riesgo }\end{array}$ \\
\hline \multicolumn{7}{|c|}{ Resultado prueba de esfuerzo electrocardiográfica } \\
\hline \multicolumn{2}{|c|}{ Fuertemente positivo } & Débilmente positivo & \multicolumn{2}{|c|}{ No concluyente } & & Negativo \\
\hline \multicolumn{2}{|c|}{$\begin{array}{l}\text { Depresión del ST plana o en } \\
\text { descenso } \geq 1 \mathrm{~mm} \text { y angina, } \\
\text { depresión del ST }>2 \mathrm{~mm} \text { sin } \\
\text { angina }\end{array}$} & $\begin{array}{c}\text { Depresión del ST plana o en } \\
\text { descenso < } 1 \text { mm y angina, o } \\
\text { depresión del ST entre } 1 \mathrm{~mm} \text { y } 2 \\
\mathrm{~mm} \text { sin angina }\end{array}$ & \multicolumn{2}{|c|}{$\begin{array}{l}\text { Depresión del ST plana o en } \\
\text { descenso }<1 \mathrm{~mm} \text { sin angina, pero } \\
\text { que no alcanzó la frecuencia } \\
\text { cardiaca esperada o presenta } \\
\text { limitaciones para hacer ejercicio } \\
\text { de origen no cardiaco }\end{array}$} & $\begin{array}{l}\text { Depr } \\
\text { descens } \\
\text { alcanz }\end{array}$ & $\begin{array}{l}\text { resión del ST plana o en } \\
\text { so }<1 \mathrm{~mm} \text { sin angina y que } \\
z 0 \text { la frecuencia cardiaca } \\
\text { esperada }\end{array}$ \\
\hline \multicolumn{2}{|c|}{$\begin{array}{l}\text { Probable enfermedad } \\
\text { coronaria. } \\
\text { Se indica angiografía } \\
\text { coronaria directa si los } \\
\text { síntomas persisten o el } \\
\text { pronóstico es de alto riesgo }\end{array}$} & \multicolumn{3}{|c|}{$\begin{array}{l}\text { Utilizar otros métodos diagnósticos como prueba de estrés } \\
\text { con imágenes, angiografía coronaria por tomografía } \\
\text { computarizada o angiografía coronaria según las } \\
\text { características y preferencias del paciente }\end{array}$} & Enfe & $\begin{array}{l}\text { improbable. } \\
\text { derar otras causas de } \\
\text { dolor torácico. }\end{array}$ \\
\hline
\end{tabular}

Figura 8. Diagnóstico de la enfermedad coronaria estable. Tomado y adaptado de "20। 3 ESC guidelines on the management of stable coronary artery disease: the Task Force on the management of stable coronary artery disease of the European Society of Cardiology" por G Montalescot y colaboradores, 20 I3, Eur Heart J, 34, p. 2949-3003. Copyright ${ }^{\circledR}$ 2015 por European Society of Cardiology.

\section{Criterios electrocardiográficos que pueden mejoran las características operativas de la prueba de esfuerzo electrocardiográfica}

Teniendo en cuenta las características operativas de la prueba de esfuerzo electrocardiográfica para el diagnóstico de enfermedad coronaria estable se ha intentado en los últimos años mejorar su rendimiento diagnóstico, con la incorporación adicional de información que incluya nuevos criterios y combinaciones de hallazgos más allá del segmento ST [6]. Para esto es necesario considerar los efectos de la isquemia inducida por el ejercicio en el electrocardiograma. Se 
ha descrito que la forma como responde la frecuencia cardíaca durante el esfuerzo físico [23], los cambios en la duración del complejo QRS [24,25], la amplitud del QRS [26], la duración del intervalo QT [27], la dispersión del QT y los subintervalos de la repolarización (duración desde el pico hasta el final de la onda T) [28] podrían mejoran la capacidad diagnóstica de la prueba de esfuerzo electrocardiográfica.

\section{Utilidad de la prueba de esfuerzo electrocardiográfica en el pronóstico de la enfermedad coronaria estable}

Es importante diferenciar la utilidad que puede tener la prueba de esfuerzo electrocardiográfica en el diagnóstico o la identificación de individuos con enfermedad coronaria en el presente de la capacidad que tiene la prueba de predecir eventos coronarios y mortalidad en el futuro. Es común que en la prueba de esfuerzo electrocardiográfica la exactitud diagnóstica se evalúe de manera separada de la exactitud pronóstica. Mientras que la exactitud diagnóstica puede ser evaluada solamente en una minoría de pacientes, a quienes posteriormente se les realiza una coronariografía (el estándar de oro), la exactitud pronóstica puede ser evaluada en un grupo de personas a quienes se sigue en el tiempo para la detección de algunos desenlaces clínicos (o estándar de comparación).

Luego de realizar el diagnóstico de enfermedad coronaria estable, el paso siguiente es la estratificación del riesgo de muerte para establecer el pronóstico, lo cual permite identificar aquellos pacientes a quienes se les debe realizar una angiografía coronaria y una revascularización si la anatomía coronaria es apropiada. A partir de algunos estudios de cohorte, en los cuales se ha obtenido información de los desenlaces a largo plazo en el seguimiento, se ha definido que los pacientes con síntomas debidos a enfermedad coronaria estable, que tienen compromiso del tronco principal izquierdo o enfermedad de tres vasos, la revascularización coronaria tiene un mejor pronóstico que la terapia médica óptima, particularmente cuando hay un deterioro de la función ventricular sistólica [2].

En el contexto de una enfermedad coronaria estable un riesgo bajo se define por una mortalidad a un año menor que el $1 \%$, un riesgo intermedio del $1 \%$ al 3\% y un riesgo alto mayor que el 3\%. El valor pronóstico del cálculo de riesgo de muerte a un año en presencia de enfermedad coronaria estable está influenciado por la historia clínica (edad, antecedente de tabaquismo, entre otros), la fracción de eyección ventricular izquierda, la respuesta a la prueba de esfuerzo electrocardiográfica y la anatomía coronaria [29]. El cálculo de la probabilidad de muerte a un año se puede realizar con algoritmos que incluyen información clínica y derivada de la prueba de esfuerzo electrocardiográfica, como el puntaje en banda rodante de Duke, que combina la duración del ejercicio, el grado de depresión del segmento ST y la severidad de la angina de pecho durante la prueba [29-32].

Lauer y colaboradores, en 2007, publicaron un algoritmo que tiene una mejor capacidad de discriminación y calibración que el puntaje en banda rodante de Duke, el cual incluye variables demográficas (edad y sexo), clínicas (historia de tabaquismo, hipertensión, diabetes y angina típica) y hallazgos derivados de la prueba de esfuerzo electrocardiográfica (capacidad funcional, cambios 
en el segmento ST, síntomas, recuperación de la frecuencia cardíaca y extrasístoles ventriculares en la recuperación) [33] (véase figura 8). El cálculo de esta probabilidad es recomendado por las guías de la Sociedad Europea de Cardiología [2] y se relaciona con la severidad angiográfica de la enfermedad coronaria. Los pacientes con un puntaje de alto riesgo tienen una mayor probabilidad de presentar una enfermedad coronaria de tres vasos o compromiso del tronco principal izquierdo, lo cual también puede ayudar a definir la estrategia de tratamiento de estos pacientes.

Como se mencionó anteriormente, el valor pronóstico dela prueba de esfuerzo electrocardiográfica en la predicción de la mortalidad total y cardiovascular se ha mejorado con la inclusión de información demográfica, clínica y derivada de la prueba de esfuerzo electrocardiográfica, a partir de la obtención de puntajes que combinan dichas variables [29-33]. Sin embargo, existen otras variables que se estudian durante la realización de la prueba de esfuerzo electrocardiográfica que de manera individual también pueden tener la capacidad en la predicción de los posibles desenlaces clínicos [6,8]. La capacidad funcional [34-36], las alteraciones en el control autonómico, la respuesta cronotrópica [37-39], la recuperación de la frecuencia cardíaca [4042], la variabilidad de la frecuencia cardíaca [43,44], la aparición de extrasístoles ventriculares durante el ejercicio y la recuperación [45-47], la respuesta de la presión arterial [48-5 I], la angina [30] y la depresión del segmento ST ajustada por la frecuencia cardíaca [52], se relacionan con la mortalidad total y cardiovascular [6,8,43].

En las tablas 6 y 7 se presentan dos casos clínicos que ilustran la toma de decisiones a partir de los resultados de la prueba de esfuerzo electrocardiográfica en el diagnóstico y pronóstico de la enfermedad coronaria.

\section{La prueba de esfuerzo electrocardiográfica en los servicios de urgencias}

El dolor torácico es una causa común de consulta en el servicio de urgencias en adultos, que da cuenta del 10\% de todas las visitas no relacionadas con trauma [53]. Una vez que se ha descartado un síndrome coronario agudo u otra causa de alto riesgo y el paciente ha permanecido mínimo durante seis horas de observación, que puede ser en una Unidad de Dolor Torácico (UDT) en el servicio de urgencias, se debe realizar un método diagnóstico que permita descartar la presencia de enfermedad coronaria [54]. Un resultado negativo disminuye la probabilidad de enfermedad coronaria y permite un alta segura del servicio de urgencias después de un corto período de observación [55].

La prueba de esfuerzo electrocardiográfica es un método de elección en este entorno clínico para pacientes que tienen un electrocardiograma en reposo normal y la capacidad de hacer ejercicio. La prueba de esfuerzo electrocardiográfica debe realizarse antes del alta del servicio de urgencias y de no ser posible, en las siguientes 48 horas. Aunque la angiografía por tomografía computarizada es utilizada con mayor frecuencia en países como Estados Unidos para el estudio de los pacientes con dolor torácico en el servicio de urgencias, no hay suficiente evidencia sobre el costo-efectividad; además, es necesario tener en cuenta que se expone a las personas al riesgo de la radiación [56-58]. 
Tabla 6. Caso clínico de enfermedad coronaria Información antes de la prueba de esfuerzo electrocardiográfica

Mujer de 65 años, quien consulta por episodio de angina atípica. Tiene antecedente de hipertensión arterial. Probabilidad pretest de enfermedad coronaria: $28 \%$ (intermedia)

\section{Información de la prueba de esfuerzo electrocardiográfica}

Durante la prueba se observó una depresión del segmento ST de $3 \mathrm{~mm}$ en las derivaciones V4, V5 y V6, asociado a angina típica a los cuatro minutos en el protocolo de Bruce.

Presentó una inadecuada recuperación de la frecuencia cardíaca y múltiples extrasístoles ventriculares luego de finalizado el ejercicio.

Interpretación de la prueba de esfuerzo electrocardiográfica

Prueba positiva para la inducción de isquemia

La prueba de esfuerzo electrocardiográfica tiene una razón de probabilidad positiva de 6

Probabilidad postest de enfermedad coronaria: $70 \%$

Riesgo de muerte a un año: 3,1\% (alto)

\section{Comentarios}

Por ser una prueba positiva, en presencia de una probabilidad postest alta y un riesgo de muerte a un año alto, se recomienda realizar coronariografía.

\footnotetext{
Tabla 7. Caso clínico negativo para enfermedad coronaria

Información antes de la prueba de esfuerzo electrocardiográfica

Paciente masculino de 55 años, que consulta por dolor no anginoso. No tiene antecedentes cardiovasculares de importancia.

Probabilidad pretest de enfermedad coronaria: 34\% (intermedia)

\section{Información de la prueba de esfuerzo electrocardiográfica}

Durante la prueba no se observó depresión del segmento ST, no presentó angina, y alcanzó 10 minutos en el protocolo de Bruce. No presentó arritmias y la recuperación de la frecuencia cardíaca fue adecuada.

\section{Interpretación de la prueba de esfuerzo electrocardiográfica}

Prueba negativa para la inducción de isquemia

La prueba de esfuerzo electrocardiográfica tiene una razón de probabilidad negativa de 0,4

Probabilidad postest de enfermedad coronaria: I8\%

Riesgo de muerte a un año: I\% (bajo)

\section{Comentarios}

Por ser una prueba negativa, en presencia de una probabilidad postest baja y un riesgo de muerte a un año bajo, la enfermedad coronaria es poco probable. Se recomienda buscar otras causas de dolor torácico.
}

\section{Información a incluir en el reporte de una prueba de esfuerzo electrocardiográfica}

La información que se obtiene de la prueba de esfuerzo electrocardiográfica debe ser recogida, registrada y almacenada adecuadamente. El reporte debe contener los datos necesarios para que el médico que solicita la prueba tome la mejor decisión clínica, además cumple un doble propósito:

a. Proporcionar información técnica sobre el desarrollo de la prueba que contenga los datos sobre los diagnósticos previos, situación clínica y preparación previa del paciente; además, el protocolo utilizado, los datos obtenidos antes, durante y después de la prueba.

b. Ofrecer una información concreta sobre el resultado de la prueba que incluya tanto los hallazgos que tienen implicaciones en el diagnóstico como en el pronóstico del paciente. Teniendo en cuenta los avances recientes, el reporte de la prueba de esfuerzo no debería enfocarse sólo en clasificarla como negativa o positiva según los hallazgos en el segmento ST [6] (véase tabla 8). 


\section{Conclusiones}

\begin{tabular}{|c|c|}
\hline Componente & Descripción \\
\hline $\begin{array}{l}\text { Probabilidad pretest de enfermedad } \\
\text { coronaria }\end{array}$ & Alta/intermedia/baja \\
\hline $\begin{array}{l}\text { Respuesta del segmento ST al ejercicio } \\
\text { y aparición de angina }\end{array}$ & Negativa/positiva/no concluyente \\
\hline $\begin{array}{l}\text { Probabilidad postest de enfermedad } \\
\text { coronaria }\end{array}$ & Alta/intermedia/baja \\
\hline Capacidad funcional & Alto/bajo riesgo de mortalidad total \\
\hline Respuesta cronotrópica & $\begin{array}{l}\text { Alto/bajo riesgo de mortalidad } \\
\text { Anormal cuando es menor o igual que el } 80 \% \text { de la frecuencia cardiaca } \\
\text { de reserva para quienes no toman } \beta \text {-bloqueadores y menor o igual que } \\
\text { el } 62 \% \text { para quienes toman } \beta \text {-bloqueadores }\end{array}$ \\
\hline Recuperación de la frecuencia cardíaca & Alto/bajo riesgo de mortalidad \\
\hline $\begin{array}{l}\text { Extrasístoles ventriculares durante la } \\
\text { recuperación }\end{array}$ & Alto/bajo riesgo de mortalidad \\
\hline $\begin{array}{l}\text { Puntaje de Duke en banda rodante [30] } \\
\text { Puntaje de Lauer [33] }\end{array}$ & Alto/intermedio/bajo riesgo de mortalidad total a un año \\
\hline \multicolumn{2}{|c|}{$\begin{array}{l}\text { Tomado y adaptado de "Exercise electrocardiogram testing: beyond the ST segment" por P. Kligfield y MS Lauer. 2006, } \\
\text { Circulation, I I 4, p. 2075. Copyright (C) } 2015 \text { por American Heart Association, Inc.. }\end{array}$} \\
\hline
\end{tabular}

La prueba de esfuerzo electrocardiográfica se encuentra entre los métodos de más fácil acceso y menos costo para la evaluación diagnóstica y pronóstica de la enfermedad coronaria. Si bien no tiene la exactitud de otros métodos diagnósticos no invasivos con imágenes aún no ha sido sustituida por estos nuevos métodos. Sin embargo, para su mejor utilización se debe tener en cuenta que el paciente tenga un electrocardiograma en reposo normal y capacidad para hacer ejercicio, como caminar con seguridad en una banda rodante. La prueba de esfuerzo electrocardiográfica sigue siendo un método diagnóstico adecuado para el estudio de los pacientes con síntomas estables que tienen un riesgo bajo o intermedio de enfermedad coronaria y en aquellas personas con dolor torácico que consultan al servicio de urgencias, en quienes se descarta un síndrome coronario agudo u otras condiciones clínicas graves. Además, si se utilizan acertadamente los análisis probabilísticos, antes y después de realizar la prueba su utilidad clínica se fortalece y la toma de decisiones resulta más adecuada.

\section{Bibliografía}

I. Fletcher GF, Ades PA, Kligfield P, Arena R, Balady GJ, Bittner VA, et al. Exercise standards for testing and training: a scientific statement from the American Heart Association. Circulation 2013; 128: 873-934.

2. Montalescot G, Sechtem U, Achenbach S, Andreotti F, Arden C, Budaj A, et al. 2013 ESC guidelines on the management of stable coronary artery disease: the Task Force on the management of stable coronary artery disease of the European Society of Cardiology. Eur Heart J 20 I3; 34: 2949-3003.
3. Bousfield G. Angina pectoris: Changes in electrocardiogram during paroxysm. Lancet 19|8; 2: 457.

4. Feil H, Siegel M. Electrocardiographic changes during attacks of angina pectoris. Am J Med Sci 1928; 175: 225.

5. Gibbons RJ, Balady GJ, Bricker JT, Chaitman BR, Fletcher GF, Froelicher VF, et al. ACC/AHA 2002 guideline update for exercise testing: summary article: a report of the American College of Cardiology/ American Heart Association Task Force on Practice 
Guidelines (Committee to Update the 1997 Exercise Testing Guidelines). Circulation 2002; 106: | 883- 1892.

6. Kligfield P, Lauer MS. Exercise electrocardiogram testing: beyond the ST segment. Circulation 2006; I 14: 2070-2082

7. Higgins JP, Higgins JA. Electrocardiographic exercise stress testing: an update beyond the ST segment. Int | Cardiol 2007; | | 6: 285-299.

8. Miller TD. The exercise treadmill test: estimating cardiovascular prognosis. Cleve Clin J Med 2008; 75: 424-430.

9. Kligfield P, Okin PM. Evolution of the exercise electrocardiogram. Am J Cardiol 1994; 73: 1209-1210.

10. Myerburg RJ, Chaitman BR, Ewy GA, Lauer MS. Task force 2: training in electrocardiography, ambulatory electrocardiography, and exercise testing. J Am Coll Cardiol 2008; 51: 348-354.

II. Rich JD, Chen S, Ward RP. Comparison of high risk stress myocardial perfusion imaging findings in men with rapid versus prolonged recovery of ST-segment depression after exercise stress testing. Am J Cardiol 2010; 105: |36|-1364.

12. Sapin PM, Koch G, Blauwet MB, McCarthy JJ, Hinds SW, Gettes LS. Identification of false positive exercise tests with use of electrocardiographic criteria: a possible role for atrial repolarization waves. J Am Coll Cardiol 1991; |8: 127-135.

13. Gussak I, Wright RS, Kopecky SL, Hammill SC. Exercise-induced ST segment elevation in $\mathrm{Q}$ wave leads in postinfarction patients: defining its meaning and utility in today's practice. Cardiology 2000; 93: 205-209.

14. Albers AR, Krichavsky MZ, Balady GJ. Stress testing in patients with diabetes mellitus: diagnostic and prognostic value. Circulation 2006; 113: 583-592.

15. Jaimes F. Pruebas diagnósticas: uso e interpretación. Acta Med Colomb 2007; 32: 29-33.

16. Genders TS, Steyerberg EW, Hunink MG, Nieman K, Galema TW, Mollet NR, et al. Prediction model to estimate presence of coronary artery disease: retrospective pooled analysis of existing cohorts. BMJ 2012; 344: e3485.

17. Gianrossi R, Detrano R, Mulvihill D, Lehmann K, Dubach P, Colombo A, et al. Exercise-induced ST depression in the diagnosis of coronary artery disease. A meta-analysis. Circulation 1989; 80: 87-98.

18. Bayes T. An essay towards solving a problem in the doctrine of chances. Philosophical Transactions of the Royal Society 1763; 53: 370-4I8.

19. Fagan TJ. Letter: Nomogram for Bayes theorem. $N$ Engl J Med 1975; 293: 257.

20. Caraguel CG, Vanderstichel R. The two-step Fagan's nomogram: ad hoc interpretation of a diagnostic test result without calculation. Evid Based Med 2013; 18: 125-128.
21. Qaseem A, Fihn SD, Williams S, Dallas P, Owens DK, Shekelle P. Diagnosis of stable ischemic heart disease: summary of a clinical practice guideline from the American College of Physicians/American College of Cardiology Foundation/American Heart Association/American Association for Thoracic Surgery/Preventive Cardiovascular Nurses Association/ Society of Thoracic Surgeons. Ann Intern Med 2012; I57: 729-734.

22. Douglas PS, Hoffmann U, Patel MR, Mark DB, AIKhalidi HR, Cavanaugh B, et al. Outcomes of anatomical versus functional testing for coronary artery disease. N Engl J Med 20 I5; 372: |29|- 300.

23. Okin PM, Kligfield P. Heart rate adjustment of ST segment depression and performance of the exercise electrocardiogram: a critical evaluation. J Am Coll Cardiol 1995; 25: 1726-1735.

24. Michaelides A, Ryan JM, VanFossen D, Pozderac R, Boudoulas $\mathrm{H}$. Exercise-induced QRS prolongation in patients with coronary artery disease: a marker of myocardial ischemia. Am Heart J 1993; 126: 1320 1325.

25. Conti A, Alesi A, Aspesi G, Bigiarini S, Bianchi S, Angeli $\mathrm{E}$, et al. Comparison of exercise electrocardiogram and exercise echocardiography in intermediate-risk chest pain patients. Am J Emerg Med 2015; 33: 7-13.

26. Bonoris PE, Greenberg PS, Christison GW, Castellanet MJ, Ellestad MH. Evaluation of R wave amplitude changes versus ST-segment depression in stress testing. Circulation 1978; 57: 904-910.

27. Lax KG, Okin PM, Kligfield P. Electrocardiographic repolarization measurements at rest and during exercise in normal subjects and in patients with coronary artery disease. Am Heart J 1994; 128: 27|-280.

28. Yu PN, Bruce RA, Lovejoy FW, Pearson R. Observations on the Change of Ventricular Systole (Qt Interval) during Exercise. J Clin Invest 1950; 29: 279-289.

29. Fearon WF, Gauri AJ, Myers J, Raxwal VK, Atwood JE, Froelicher VF. A comparison of treadmill scores to diagnose coronary artery disease. Clin Cardiol 2002; 25: 117-122

30. Mark DB, Hlatky MA, Harrell FE, Jr., Lee KL, Califf RM, Pryor DB. Exercise treadmill score for predicting prognosis in coronary artery disease. Ann Intern Med 1987; 106: 793-800.

31. Morise AP, Jalisi F. Evaluation of pretest and exercise test scores to assess all-cause mortality in unselected patients presenting for exercise testing with symptoms of suspected coronary artery disease. J Am Coll Cardiol 2003; 42: 842-850.

32. Hesse B, Morise A, Pothier CE, Blackstone EH, Lauer MS. Can we reliably predict long-term mortality after exercise testing? An external validation. Am Heart J 2005; 150: 307-3।4.

33. Lauer MS, Pothier CE, Magid DJ, Smith SS, Kattan MW. An externally validated model for predicting long-term survival after exercise treadmill testing in 
patients with suspected coronary artery disease and a normal electrocardiogram. Ann Intern Med 2007; 147: $821-828$

34. Blair SN, Kohl HW, 3rd, Paffenbarger RS, Jr., Clark DG, Cooper KH, Gibbons LW. Physical fitness and all-cause mortality. A prospective study of healthy men and women. JAMA 1989; 262: 2395-2401.

35. Wei M, Kampert JB, Barlow CE, Nichaman MZ, Gibbons LW, Paffenbarger RS, Jr., et al. Relationship between low cardiorespiratory fitness and mortality in normal-weight, overweight, and obese men. JAMA 1999; 282: I547-| 553.

36. Myers J, Prakash M, Froelicher V, Do D, Partington S, Atwood JE. Exercise capacity and mortality among men referred for exercise testing. $N$ Engl J Med 2002; 346: 793-80I.

37. Lauer MS, Okin PM, Larson MG, Evans JC, Levy D. Impaired heart rate response to graded exercise. Prognostic implications of chronotropic incompetence in the Framingham Heart Study. Circulation 1996; 93: 1520-1526.

38. Lauer MS, Francis GS, Okin PM, Pashkow FJ, Sna$\operatorname{der}$ CE, Marwick TH. Impaired chronotropic response to exercise stress testing as a predictor of mortality. JAMA 1999; 281: 524-529.

39. Myers J, Tan SY, Abella J, Aleti V, Froelicher VF. Comparison of the chronotropic response to exercise and heart rate recovery in predicting cardiovascular mortality. Eur J Cardiovasc Prev Rehabil 2007; 14: 215-221.

40. Cole CR, Foody JM, Blackstone EH, Lauer MS. Heart rate recovery after submaximal exercise testing as a predictor of mortality in a cardiovascularly healthy cohort. Ann Intern Med 2000; I 32: 552-555.

4I. Cheng YJ, Lauer MS, Earnest CP, Church TS, Kampert JB, Gibbons LW, et al. Heart rate recovery following maximal exercise testing as a predictor of cardiovascular disease and all-cause mortality in men with diabetes. Diabetes Care 2003; 26: 2052-2057.

42. Lipinski MJ, Vetrovec GW, Froelicher VF. Importance of the first two minutes of heart rate recovery after exercise treadmill testing in predicting mortality and the presence of coronary artery disease in men. Am J Cardiol 2004; 93: 445-449.

43. Heart rate variability: standards of measurement, physiological interpretation and clinical use. Task Force of the European Society of Cardiology and the North American Society of Pacing and Electrophysiology. Circulation 1996; 93: $1043-1065$.

44. Dewey FE, Freeman JV, Engel G, Oviedo R, Abrol $\mathrm{N}$, Ahmed N, et al. Novel predictor of prognosis from exercise stress testing: heart rate variability response to the exercise treadmill test. Am Heart J 2007; 153: 28|-288.

45. Beckerman J, Wu T, Jones S, Froelicher VF. Exercise test-induced arrhythmias. Prog Cardiovasc Dis 2005; 47: 285-305.
46. Jouven X, Zureik M, Desnos M, Courbon D, Ducimetiere $\mathrm{P}$. Long-term outcome in asymptomatic men with exercise-induced premature ventricular depolarizations. N Engl J Med 2000; 343: 826-833.

47. Frolkis JP, Pothier CE, Blackstone EH, Lauer MS. Frequent ventricular ectopy after exercise as a predictor of death. N Engl J Med 2003; 348: 78I-790.

48. Dubach P, Froelicher VF, Klein J, Oakes D, GroverMcKay M, Friis R. Exercise-induced hypotension in a male population. Criteria, causes, and prognosis. Circulation 1988; 78: 1380-1387.

49. Hammermeister KE, DeRouen TA, Dodge HT, Zia M. Prognostic and predictive value of exertional hypotension in suspected coronary heart disease. Am J Cardiol 1983; 51: 1261-1266.

50. Mundal R, Kjeldsen SE, Sandvik L, Erikssen G, Thaulow E, Erikssen J. Exercise blood pressure predicts cardiovascular mortality in middle-aged men. Hypertension 1994; 24: 56-62.

5I. Allison TG, Cordeiro MA, Miller TD, Daida H, Squires RW, Gau GT. Prognostic significance of exercise-induced systemic hypertension in healthy subjects. Am J Cardiol 1999; 83: 37।-375.

52. Okin PM, Anderson KM, Levy D, Kligfield P. Heart rate adjustment of exercise-induced ST segment depression. Improved risk stratification in the Framingham Offspring Study. Circulation 1991; 83: 866-874.

53. Bhuiya FA, Pitts SR, McCaig LF. Emergency department visits for chest pain and abdominal pain: United States, 1999-2008. NCHS Data Brief 20 I0: I-8.

54. Aristizábal D, Montoya C, Valencia A, Jaime J, Gallo J. Evaluación del impacto clínico y la seguridad de una unidad de dolor torácico en pacientes con probabilidad baja e intermedia de síndrome coronario agudo. Rev Colomb Cardiol 2015.

55. Amsterdam EA, Kirk JD, Bluemke DA, Diercks D, Farkouh ME, Garvey JL, et al. Testing of low-risk patients presenting to the emergency department with chest pain: a scientific statement from the American Heart Association. Circulation 20 I0; 122: 1756- 1776.

56. Zeb I, Abbas N, Nasir K, Budoff MJ. Coronary computed tomography as a cost-effective test strategy for coronary artery disease assessment - a systematic review. Atherosclerosis 2014; 234: 426-435.

57. Thomas DM, Shaw DJ, Barnwell ML, Jones RL, Ahmadian HR, Prentice RL, et al. The lack of obstructive coronary artery disease on coronary CT angiography safely reduces downstream cost and resource utilization during subsequent chest pain presentations. J Cardiovasc Comput Tomogr 2015; 9: 329-336.

58. Genders TS, Petersen SE, Pugliese F, Dastidar AG, Fleischmann KE, Nieman K, et al. The optimal imaging strategy for patients with stable chest pain: a cost-effectiveness analysis. Ann Intern Med 2015; 162: 474-484. 


\section{$\pi$ Laboratorio ${ }^{\circledR}$ Clínico U Hematológico}

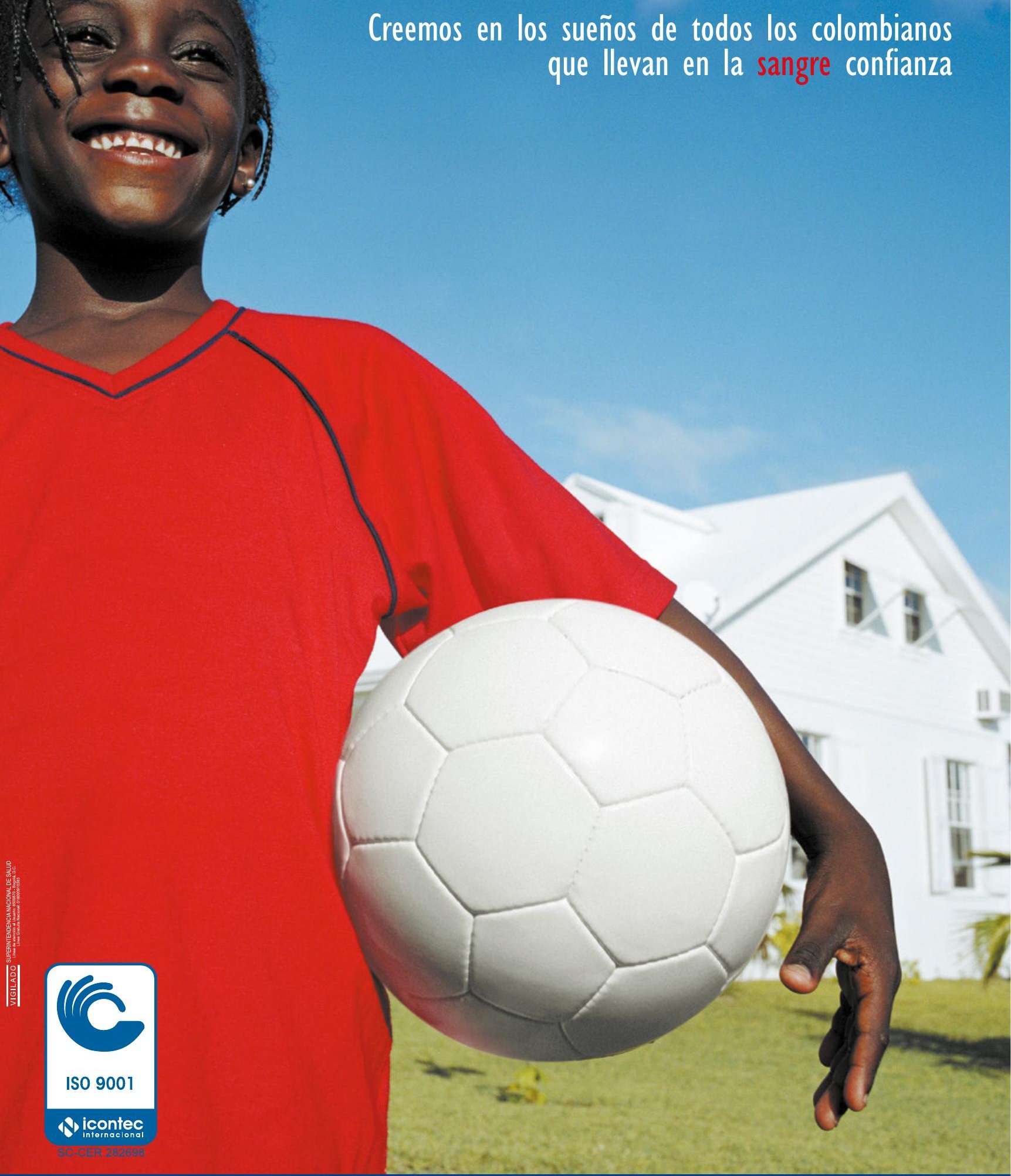

Sede Principal Poblado: Carrera 43C No. 5-33

Toma de Muestras: Centro Comercial Sandiego, Torre Norte, Piso 10, No. 1034

Teléfono: 4444200 - www.lch.co - Medellín 